\title{
Comparison and Regulation of Neuronal Synchronization for Various STDP Rules
}

\author{
Yanhua Ruan and Gang Zhao \\ Institute of Complex Bio-dynamics, Jiangxi Blue Sky University, Nanchang, Jiangxi 330098, China \\ Correspondence should be addressed to Gang Zhao, zhg2601@ustc.edu
}

Received 22 August 2008; Revised 28 January 2009; Accepted 5 May 2009

Recommended by Menahem Segal

\begin{abstract}
We discuss effects of various experimentally supported STDP learning rules on frequency synchronization of two unidirectional coupled neurons systematically. First, we show that synchronization windows for all STDP rules cannot be enhanced compared to constant connection under the same model. Then, we explore the influence of learning parameters on synchronization window and find optimal parameters that lead to the widest window. Our findings indicate that synchronization strongly depends on the specific shape and the parameters of the STDP update rules. Thus, we give some explanations by analyzing the synchronization mechanisms for various STDP rules finally.
\end{abstract}

Copyright (c) 2009 Y. Ruan and G. Zhao. This is an open access article distributed under the Creative Commons Attribution License, which permits unrestricted use, distribution, and reproduction in any medium, provided the original work is properly cited.

\section{Introduction}

Synchronous activity is a basic characteristic in the brain. It exists in many regions of the brain, such as CA1 of the hippocampus [1], visual cortex [2] and cortical areas correlating with conscious perception [3]. It is known that synchronization is very important for information processing, such as predicting sensory input [4], and information codes [5]. Moreover, synchronous activity plays a crucial role in epileptic activity $[6,7]$, modulation of neurons about attention [8], memory and learning $[9,10]$, and cognitive functions [11].

Since the discovery of long term potentiation (LTP) and LTD (long term depression) [12-14], it has been debated how synaptic modifications are correlated to neuron activities. Spike timing-dependent plasticity (STDP) is a form of synaptic modification discovered relatively recently, which depends on the relative timing of pre- and post-synaptic action potentials at a millisecond time scale $[15,16]$. Many experiments have proved the existence of STDP, such as in neocortical slices [17], hippocampus slice [18], hippocampal cell cultures [19], and tadpole rectum in vivo [20]. In addition, STDP provides powerful mechanisms for models of temporal pattern recognition [21], temporal sequence learning $[22,23]$, a continuous-time associative memory
[24], coincidence detection [16, 25], navigation [26, 27] and direction selectivity [28].

The interaction among neurons relies much on synaptic modification in which STDP is the only one that greatly expands the capability of Hebbian learning to address temporally sensitive computational tasks. STDP in synchronization has attracted wide interests. For example, the result of learning-induced synchronization of a neural network at various developing stages using STDP rule is consistent with recent experimental observations [29]. Furthermore, the comparison of synchronization between discontinuous antiSTDP(dc-aSTDP, see Section 2) and constant connection has been investigated [30]. Following it, the continuous STDP(cSTDP, see Section 2) has also been studied [31] by the same authors. They suggest that a functional role of STDP might be enhancing synchronization. Motivated by their work, we systemically discuss the roles of four types of STDP rules(cSTDP, dc-STDP, dc-aSTDP and in-STDP, see Section 2) in frequency synchronization in the present paper, employing the same model [31] with only values of some parameters different, such as $A_{\text {plus }}, t_{\text {syn }}, V_{\text {slope }}, g_{\max }$ (see Section 3 ).

We find, however, not all STDP rules facilitate synchronization. It encourages us to trace the reason. We then consider if the learning curves, which characterize the STDP rule, have certain effects on synchronization. 
Results indicate that synchronization strongly depends on the specific shape and the parameters of the STDP rule. However, the optimal synchronization ranges for dc-STDP and in-STDP, got from regulating learning parameters, are not wider than those for the corresponding strongest constant connection respectively. As a result, when we seek the reason, we discover that the synchronization mechanisms of above four STDP rules can be classified into two categories: (i) c-STDP and dc-aSTDP rules; (ii) dc-STDP and inSTDP rules. The synchronization mechanisms of the two categories are different. For c-STDP and dc-aSTDP rules, two neurons' synchronization either relies on the balancing out potentiation and depression during one cycle consistent with the perspective of Nowotny et al. or relies on the maximal synaptic conductance. However, for dc-STDP and in-STDP rules, the synchronization windows are completely provided by the respective maximal synaptic conductance. As regards this finding, we offer an intuitive explanation finally.

\section{Models and Method}

We consider two HH neurons with unidirectional activitydependent excitatory or inhibitory synaptic coupling. Although such a configuration is too simple to find applications in brain information processing, it serves as a staring point for many model researches. The neurons are modeled with standard $\mathrm{Na}, \mathrm{K}$, and "leak" currents [32],

$$
\begin{aligned}
C \frac{d V_{i}(t)}{d t}= & -g_{\mathrm{Na}} \cdot m_{i}(t)^{3} \cdot h_{i}(t) \cdot\left(V_{i}(t)-E_{\mathrm{Na}}\right)-g_{\mathrm{K}} \cdot n_{i}(t)^{4} \\
& \cdot\left(V_{i}(t)-E_{\mathrm{K}}\right)-g_{L} \cdot\left(V_{i}(t)-E_{L}\right)-I_{\mathrm{syn}}(t)+I_{\mathrm{stim}},
\end{aligned}
$$

where $i=1,2$.

Each of the activation and inactivation variables $y_{i}(t)=$ $\left\{n_{i}(t), m_{i}(t), h_{i}(t)\right\}, i=1,2$ satisfies first-order kinetics,

$$
\frac{d y_{i}(t)}{d t}=\alpha_{y}\left[V_{i}(t)\right]\left[1-y_{i}(t)\right]-\beta_{y}\left[V_{i}(t)\right] y_{i}(t), \quad i=1,2 .
$$

The parameters in these equations are given in [31],

$$
\begin{aligned}
& \alpha_{n}=\frac{0.032(-50-V)}{\exp ((-50-V) / 5)-1}, \\
& \beta_{n}=0.5 \exp ((-55-V) 40), \\
& \alpha_{m}=\frac{0.32(-52-V)}{\exp ((-50-V) / 4)-1}, \\
& \beta_{m}=\frac{0.28(25+V)}{\exp ((25+V) / 5)-1}, \\
& \alpha_{h}=0.128 \exp \left(\frac{-48-V}{18}\right), \\
& \beta_{h}=\frac{4}{\exp ((-25-V) / 5)+1} .
\end{aligned}
$$

$I_{\text {stim }}$ is a constant input current forcing each neuron to spike with a constant, $I_{\text {stim }}$-dependent period, labeled as $T_{1}$ and $T_{2}$. The postsynaptic neuron would show another firing period $T_{2}{ }^{1}$, when it is driven by the synaptic current, which is dependent on the postsynaptic potential $V_{2}(t)$, the reversal potential $V_{\text {rev }}$, the activation variable $S(t)$ and its maximal conductance $g(t)$,

$$
I_{\text {syn }}(t)=g(t) S(t)\left(V_{2}(t)-V_{\text {rev }}\right),
$$

where

$$
\begin{aligned}
\frac{d S(t)}{d t} & =\frac{S_{\infty}\left(V_{1}(t)\right)-S(t)}{t_{\text {syn }} \cdot 1-\left(S_{\infty}\left(V_{1}(t)\right)\right)}, \\
S_{\infty}(V) & = \begin{cases}\tanh \left(\frac{V-V_{t h}}{V_{\text {slope }}}\right), & \text { for } V>V_{t h}, \\
0, & \text { otherwise. }\end{cases}
\end{aligned}
$$

The time-dependent synaptic coupling strength $g(t) \mathrm{nS}$ is

$$
g(t)=\frac{g_{\max }}{2}\left(\tanh \left(\frac{g_{\text {raw }}-g_{\text {mid }}}{g_{\text {slope }}}\right)+1\right) .
$$

Therefore $g(t)$ always have values between $0 \mathrm{nS}$ and $g_{\max }$. The bound imposed on $g(t)$ is artificially set to avoid unrealistically high synaptic conductance and negative conductance. In order to obtain biologically plausible synaptic conductance, several methods have been employed to limit the synaptic strength in literature, such as a negative total integral [33], artificial bounds [34], and self-limitation [31]. Unless otherwise stated, we employ the self-limitation method which is characterized by a function "tanh" in our simulation.

$g_{\text {raw }}$ is modified by STDP rules that are introduced in the next paragraph. The initial value of $g_{\text {raw }}$ is $20 \mathrm{nS}$. The parameters of the model are

$$
\begin{gathered}
C=30 \mu \mathrm{F}, \quad g_{L}=1 \mu \mathrm{S}, \quad E_{L}=-64 \mathrm{mV}, \\
g_{\mathrm{Na}}=360 \mu \mathrm{S}, \quad E_{\mathrm{Na}}=50 \mathrm{mV}, \quad g_{\mathrm{K}}=70 \mu \mathrm{S}, \\
E_{\mathrm{K}}=-95 \mathrm{mV}, \quad V_{t h}=-20 \mathrm{mV}, \quad t_{\text {syn }}=25 \mathrm{~ms}, \\
V_{\text {slope }}=15 \mathrm{mV}, \quad g_{\max }=25 \mathrm{nS}, \quad g_{\text {mid }}=\frac{1}{2} g_{\text {max }}, \\
g_{\text {slope }}=g_{\text {mid }}, \quad V_{\text {rev }}=20 \mathrm{mV} .
\end{gathered}
$$

The time-dependent synaptic coupling strength $g(t)$ is determined by the spike-timing of pre- and postsynaptic spikes. We consider four types of activity-dependent couplings that have been found in experiments: (1) an excitatory synapse with continuous STDP (c-STDP). There are two forms of c-STDP from two different experiments. One (Figure 1(a)) is from the recording of the neocortexlayer 5 Xenopus tectum hippocampus [31, 35], and the other is from the neocortex-layer 4 spiny stellates [36]. The latter form will not be considered here, because it introduces persistent decrease to synaptic strength that would result in none synchronization if two neurons have 


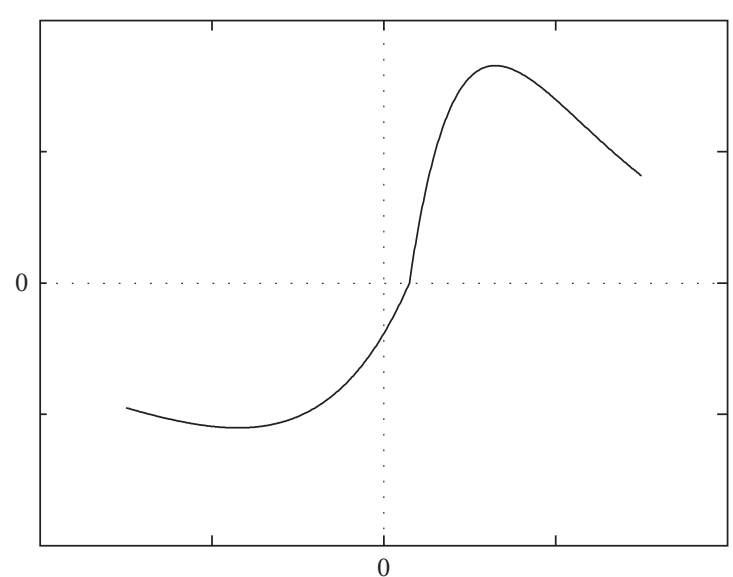

(a)

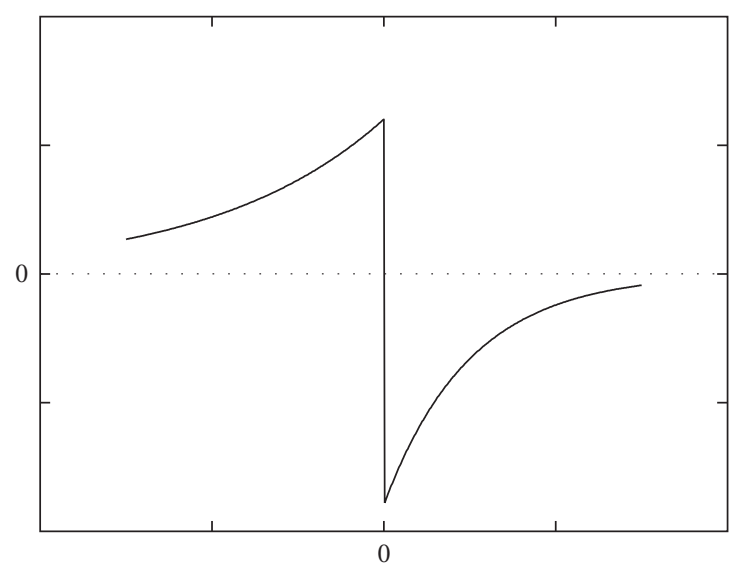

(c)

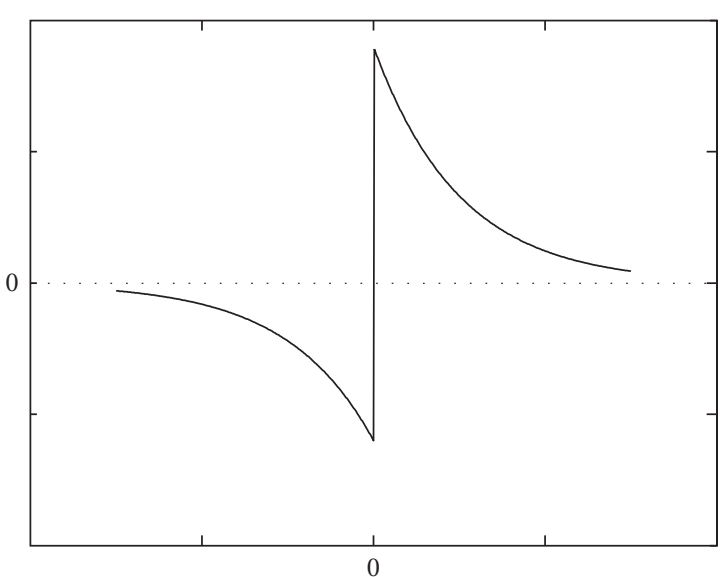

(b)

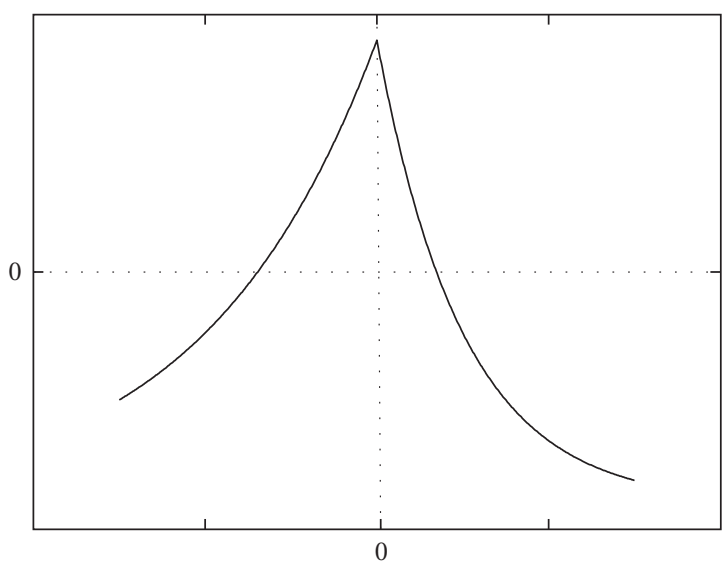

(d)

FIGURE 1: Different types of STDP curves are presented. (a) (c-STDP ) (b) discontinuous STDP(dc-STDP); (c) discontinuous anti-STDP(dcaSTDP); (d) inhibitory STDP(in-STDP).

different inherent periods. (2) an excitatory synapse with discontinuous STDP (dc-STDP, Figure 1(b)) [17, 36]; (3) an excitatory synapse with discontinuous anti-STDP [36, 37] (dc-aSTDP, Figure 1(c)); (4) an inhibitory synapse with STDP (in-STDP, Figure 1(d)) [18, 19, 36].

$\Delta g_{\text {raw }}$ is a function of $\Delta t=t_{\text {postspike }}-t_{\text {prespike }}$, time difference between the times of postsynaptic and presynaptic spikes. The learning rules corresponding to Figures 1(a), 1(b), 1(c), 1(d)) are provided as follows

c-STDP:

$$
\Delta g_{\text {raw }}= \begin{cases}A_{\text {plus }} \frac{\Delta t-\tau_{0}}{t_{\text {plus }}} \cdot e^{-\Delta t / t_{\text {plus }},} & \Delta t>\tau_{0}, \\ A_{\text {sub }} \frac{\Delta t-\tau_{0}}{t_{\text {sub }}} \cdot e^{\Delta t / t_{\text {sub }}}, & \Delta t \leq \tau_{0},\end{cases}
$$

dc-STDP:

$$
\Delta g_{\text {raw }}= \begin{cases}A_{\text {plus }} \cdot e^{-\Delta t / t_{\text {plus }},} & \Delta t>0, \\ -A_{\text {sub }} \cdot e^{\Delta t / t_{\text {sub }}}, & \Delta t \leq 0,\end{cases}
$$

dc-aSTDP:

$$
\Delta g_{\mathrm{raw}}= \begin{cases}-A_{\mathrm{plus}} \cdot e^{-\Delta t / t_{\mathrm{plus}},} & \Delta t>0, \\ A_{\mathrm{sub}} \cdot e^{\Delta t / t_{\text {sub }},} & \Delta t \leq 0,\end{cases}
$$

in-STDP:

$$
\Delta g_{\text {raw }}=\left\{\begin{array}{ll}
A_{\text {plus }} \cdot\left(e^{-\Delta t / t_{\text {plus }}}-0.5\right), & \Delta t>0, \\
A_{\text {sub }} \cdot\left(e^{\Delta t / t_{\text {sub }}}-0.5\right), & \Delta t \leq 0,
\end{array} \quad A_{\text {plus }}=A_{\text {sub }} .\right.
$$

Synchronization of pre- and post-synaptic neurons occurs when $\left|T_{1}-\left\langle T_{2}{ }^{1}\right\rangle\right|$ is limited in an acceptable range. We set the criteria of synchronization as $\left|T_{1}-\left\langle T_{2}^{1}\right\rangle\right|<$ 1.5 milliseconds. Although there is some arbitrariness in setting the criteria of synchronization, there is no qualitative change in our results if the criteria change in two folds. Each simulation runs 20 seconds, average is taken in the final 4000 milliseconds. We have observed that simulations from different initial values of $V_{2}, S$ could result in different outputs, that is, the post-synaptic neuron sometimes 
synchronizes with the pre-synaptic one, sometimes keep its original period, or sometimes fires with an oscillating period (see Figure 2(c)). We therefore carry out 40 times of simulations, from randomly selected initial values, for every $T_{2}$. The standard deviation of $\left|T_{1}-\left\langle T_{2}{ }^{1}\right\rangle\right|$, indicating how precisely the neurons are synchronized, represents the quality of synchronization. Range of $T_{2}$, in which post-synaptic neuron is successfully entrained by the pre-synaptic neuron, that is, $\left|T_{1}-\left\langle T_{2}{ }^{1}\right\rangle\right|<1.5$ in all 40 simulations, is defined as the synchronization window.

\section{Results}

3.1. Comparison of Synchronization Windows of Different Types of STDP. We investigate the width of synchronization window of various STDP curves, with the same set of parameters. The period of the pre-synaptic neuron is chosen to be 171 milliseconds, which falls into the range of theta waves. Several reasons make us choose such a long period. First, it has fairly wide synchronization windows which allow comparisons in a relative precise manner and can provide clearer information about synchronization windows of various STDP learning rules. Second, the slow theta waves always involve many neurons that fire synchronously [38, 39]. Also, theta waves have many interesting implications. For example, theta waves are normally absent in healthy awake adults, but appear during the state of meditation [40]. During emotional arousal and various types of rhythmic activities during sleep, neurons in the amygdala produce theta activity $[41,42]$. And it is known that coherent theta activity $(4-8 \mathrm{~Hz})$ in amygdala-hippocampal circuits is deeply involved in fear memory [43].

With fixed period of the pre-synaptic neuron $T_{1}$, we evaluate the coupled period of postsynaptic neuron $T_{2}{ }^{1}$ when it is driven by the pre-synaptic neuron. The values of learning parameters used in c-STDP, dc-STDP, dc-aSTDP are $A_{\text {plus }}=$ $9 \mathrm{nS}, A_{\text {sub }}=6 \mathrm{nS}, t_{\text {plus }}=100$ milliseconds, $t_{\mathrm{sub}}=200$ milliseconds, additionally $\tau_{0}=30$ milliseconds in $c-S T D P$, and in in-STDP are $A_{\text {plus }}=A_{\text {sub }}=8 \mathrm{nS}, t_{\text {plus }}=100$ milliseconds, $t_{\text {sub }}=200$ milliseconds. Our model and most values of parameters are from the model of Nowotny for c-STDP, except $A_{\text {plus }}, t_{\text {syn }}, V_{\text {slope }}, g_{\max }$ are different [31]. Especially, $T_{1}$ is fixed at 171 milliseconds in our simulations while $T_{2}$ is set to constant value 300 milliseconds of Nowotny's work.

The window of synchronization (upper panel) and quality (middle panel) of dc-STDP are presented in Figure 2 as an example. We scan $T_{2}$ from 150 milliseconds to 320 milliseconds. The upper panel shows the number of synchronization times in 40 simulations, for each $T_{2}$. It is clear that, in certain range of $T_{2}$, simulations from different initial values may have different results. Only when $T_{2}$ falls into the segment from 194 to 221, the post-synaptic neuron can synchronize with the pre-synaptic neuron from any initial value. It is easily found that there are some $T_{2}$ corresponding to the number of synchronization times between 1 and 39. In this situation, we present the three possible states of post-synaptic neuron's firing in Figure 2 (lower panel)—keeping the initial period (squares), oscillating (circles), and synchronizing

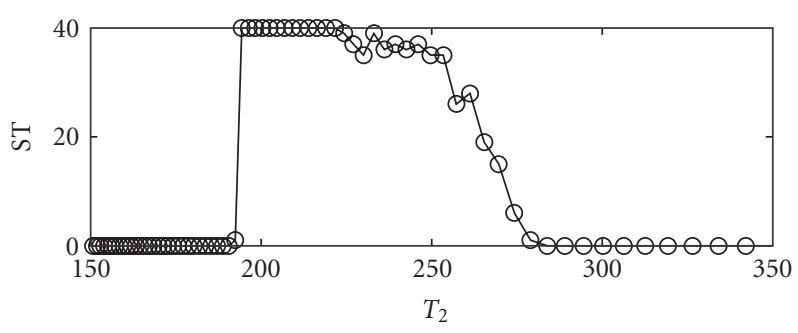

(a)

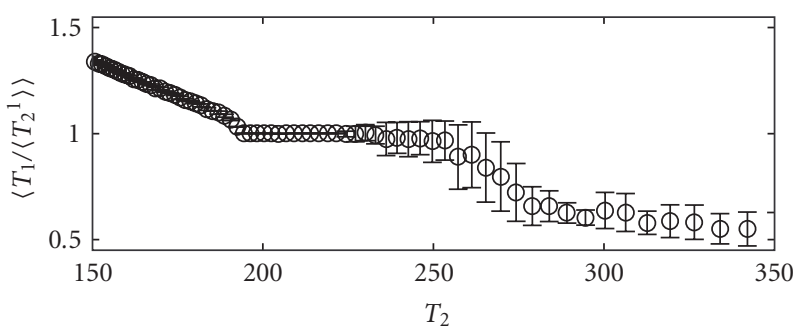

(b)

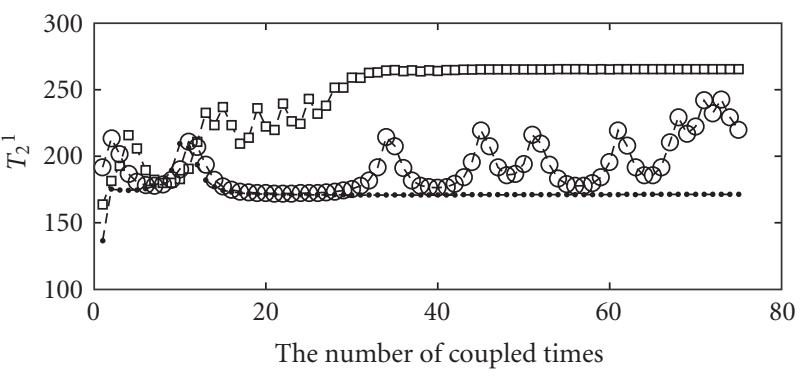

(c)

FIGURE 2: Synchronization results for dc-STDP rule carrying out 40 times. Figure 2(a) shows the number of synchronization times (ST) of fixed $T_{2}$ varying from 150 milliseconds to 320 milliseconds. Synchronization window is from 194 to 221 . The probabilistic synchronization window is from 222 to 289 . Figure 2(b) presents the quality of synchronization against the ratios of uncoupled periods. In Figure 2(c), we fix $T_{2}=265$ milliseconds which falls into the probabilistic synchronization window. There are three states of the coupled period of post-synaptic neuron $T_{2}{ }^{1}$ when we carry out 40 stimulation times: (i) keeping the initial period (squares); (ii) oscillating (circles); (iii) synchronizing with the pre-synaptic neuron (dots).

with the pre-synaptic neuron (dots). Obviously, these states are independent of the synchronization criteria we set.

For the purpose of discussing the function role of STDP rules in synchronization, the synchronization windows of various type of STDP are plotted in Figure 3, in which the case of constant synaptic conductance is also included as a comparative tool. The same parameter values used in simulations ensure a fair comparison. In our simulation studies, the synapse strength is between $0 \mathrm{nS}$ and $25 \mathrm{nS}$. We choose the maximal synaptic strength and the middle synaptic strength of STDP synapse as the synaptic strength of constant synapse in this study. Interestingly, because the synchronization windows for STDP rules are narrower than synchronization window for constant synapse $g=$ $25 \mathrm{nS}$ in Figure 3, these results, opposite to previous reports, 


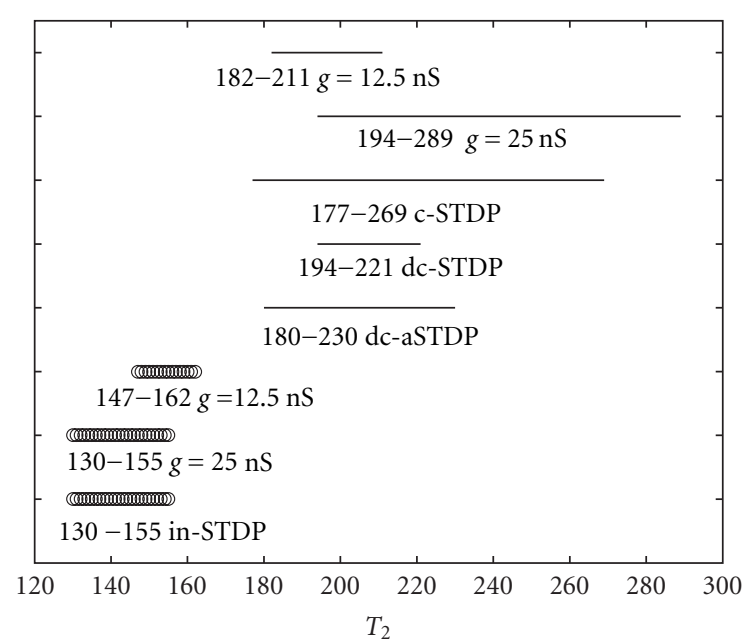

Figure 3: The synchronization window of different types of learning curves. The top five lines (points) are excitatory synapses and the bottom three lines (circles) are inhibitory synapses. See legends in the figure.

indicate that all STDP rules do not enhance synchronization comparing with the constant synapse under the chosen parameters.

Several other points are worthy of detailed describing. First, increasing the excitatory constant synaptic connections from $12.5 \mathrm{nS}$ to $25 \mathrm{nS}$, leads to a wider synchronization window. However, the wider window could not totally contain the smaller one. It extends toward larger $T_{2}$, but loses a portion of smaller $T_{2}$. The case of increasing inhibitory constant synaptic connections is alike. Second, the widest ranges of synchronization window are achieved by the excitatory constant connection $g=25 \mathrm{nS}$ and c-STDP rules. However, the lower boundary of the synchronization window of c-STDP is much nearer to $T_{1}$ than that of excitatory constant synapse. Thirdly, although c-STDP and dc-STDP are fitted from the same set of experimental data, c-STDP has a much wider synchronization window than dcSTDP.

We conclude that all STDPs do not give rise to enhanced synchronization and the window of dc-STDP is surprisingly narrow under the chosen parameters in Figure 3. Therefore, the questions about what bring about these results inspire us to study further. At the same time, Figure 2 shows a large part of probabilistic synchronization, whose range is a subset of the synchronization window of constant synaptic connection with $g=25 \mathrm{nS}$. We are interested in if the probabilistic synchronization could be enhanced into absolute synchronization by modulation of learning curves. These are the theme of the next section.

\subsection{The Effect of Learning Parameters on Synchronization.} In order to establish the functional role of STDP clearly, we consider if the learning parameters for each STDP rule have important effect on synchronization. In addition, synchrony-asynchrony transition plays important role in the brain. An increase in the degree of synchrony of a uniform input can cause transitions between memorized activity patterns in the order presented during learning. However, if synchronous input is at a low level, transitions cannot occur [44]. The synchrony-asynchrony transition have also been implemented in controlling winner-takeall competition [45], the next recalled time of associative memory [46] and the fine structure of cell assemblies [47].

In this section, we will discuss the flexibility of the synchronization window, by exploring regulation of width of the synchronization window, whose boundary indicates the synchrony-asynchrony transition. We take the modulation of learning parameters as the method to regulate the synchronization window. There are four parameters that determine a learning rule $A_{\text {plus }}, A_{\text {sub }}, t_{\text {plus }}, t_{\text {sub }}$. With three of them fixed and only one parameter changing, we could explore its influence on the width of the synchronization window. For example, $A_{\text {plus }}$ increases from an adequately small value $1 \mathrm{nS}$ to $20 \mathrm{nS}$ with a step of $1 \mathrm{nS}$. We have also scanned values that are beyond $20 \mathrm{nS}$, but find that the effect of increasing $A_{\text {plus }}$ is saturated around $20 \mathrm{nS}$. Further increasing $A_{\text {plus }}$ brings no more effect. Other parameters are fixed as: $A_{\text {sub }}=6 \mathrm{nS}$, $t_{\text {plus }}=100$ milliseconds, $t_{\text {sub }}=200$ milliseconds, $T_{2}=233$ milliseconds. In these conditions, we present the effect of $A_{\text {plus }}$ on the location of the synchronization window with the dc-STDP rule. We carry out simulations 40 times, each from different initial values.

Figure 4(a) shows the value of ARP (average change of relative period ratio $)=\left\langle T_{2}-\left\langle T_{2}{ }^{1}\right\rangle\right\rangle /\left(T_{2}-T_{1}\right)$ for different $A_{\text {plus }}$. Some points have value 0 or 1 , which means post-synaptic neuron keeping initial period or achieving synchronization with the pre-synaptic one, respectively. Some points have values other than 0 or 1 . Figure 4 (b) gives an explanation that these points correspond to probabilistic synchronizations with fixed $T_{2}=233$.

We find that the absolute synchronization range is from $10 \mathrm{nS}$ to $20 \mathrm{nS}$ in Figure 4(b). According to the definition of synchronization window of $T_{2}$, we can similarly define $10 \mathrm{nS}$ to $20 \mathrm{nS}$ as the synchronization window of $A_{\text {plus }}$, with dc-STDP rule and other fixed parameters. The boundary of this synchronization window indicates where synchronyasynchrony transition happens when changing $A_{\text {plus }}$.

From the results of Figure 4, the reason why synchronization window of constant synapse is wider than that of STDP rules (Figure 3) may be explained by learning parameters. To figure out a global picture of the effect of $A_{\text {plus }}$ on synchronization for c-STDP rule, we then determine the synchronization window of $A_{\text {plus }}$ with different $T_{2}$. $A_{\text {plus }}$ increases from $1 \mathrm{nS}$ to $20 \mathrm{nS}$ with a step of $1 \mathrm{nS}$ while other three parameters keep initial values: $A_{\text {sub }}=6 \mathrm{nS}, t_{\text {plus }}=100$ milliseconds, $t_{\mathrm{sub}}=200$ milliseconds. We choose some typical values of $T_{2}$ to character the global picture. The points in Figure 5(a) show the synchronization range of $A_{\text {plus }}$ with $T_{1}$ divided by the chosen values of $T_{2}$. The lower boundaries, as well as those upper boundaries that are other than $20 \mathrm{nS}$, indicate the position of synchrony-asynchrony transitions. For example, when $T_{2}$ is 177 milliseconds equivalent to $T_{1} / T_{2}$ $=0.966, A_{\text {plus }}$ outside of the points range from $8 \mathrm{nS}$ to $11 \mathrm{nS}$ cannot lead to synchronization between the two neurons. In addition, for those points marked on the horizontal axis, 


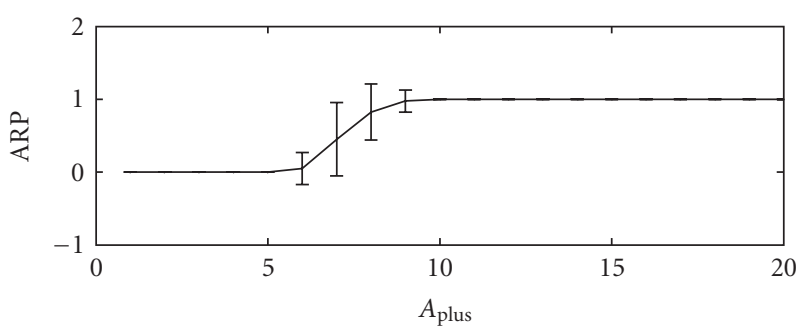

(a)

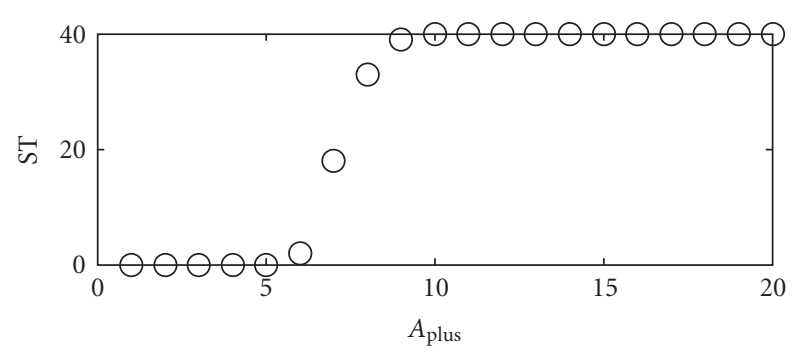

(b)

FIGURE 4: It shows that the regulation of $A_{\text {plus }}$ to synchronization for the dc-STDP carrying out 40 times of stimulations. $A_{\text {sub }}=6 \mathrm{nS}, t_{\text {plus }}$ $=100$ milliseconds, $t_{\text {sub }}=200$ milliseconds, $T_{2}=233$ milliseconds. Top: the value of ARP $=\left\langle T_{2}-\left\langle T_{2}{ }^{1}\right\rangle\right\rangle /\left(T_{2}-T_{1}\right)$ is 0 or 1 which, respectively, means post-synaptic neuron keeping initial period or achieving synchronization. Bottom: the number of synchronization times (ST) in 40 stimulations against $A_{\text {plus }}$.

synchronization could not be established no matter what values $A_{\text {plus }}$ take.

According to this global picture, the intersection of those synchronization ranges of $A_{\text {plus }}$, which is from $10 \mathrm{nS}$ to $11 \mathrm{nS}$, identifies the range of $A_{\text {plus }}$ that would lead to the widest synchronization window which is from 177 to 289 . This optimal synchronization window for c-STDP is wider than constant synapse $g=25 \mathrm{nS}$. Thus, the learning parameters strongly influence the role of STDP on synchronization.

Taking the same method as $A_{\text {plus }}$, we study the effects of other three parameters on synchronization. Figure 5 presents the situation of $A_{\text {sub }}$ varying from $1 \mathrm{nS}$ to $20 \mathrm{nS}$ with the step $1 \mathrm{nS}$ (circles, Figure 5(a)), $t_{\text {plus }}$ (points, Figure 5(b)) and $t_{\text {sub }}$ (circles, Figure 5(b)) both varying from 10 milliseconds to 400 milliseconds with the step 10 milliseconds. When changing one parameter to explore how the range of synchrony evolves with $T_{2}$, other three parameters keep their initial values as in Figure 3. Similarly, the global modulation picture of four learning parameters for other learning rules can be got. We only give the situation for c-STDP rule in Figure 5.

The optimal synchronization windows for various learning rules are presented in Figure 6 comparing with previous synchronization windows obtained in Figure 3. The parameters used for optimal synchronization windows are presented in Table 1. The parameters are derived according to regulating one parameter while other three parameters keep initial values. Under the optimal parameters, the synchronization windows for STDP rules are not narrower than constant

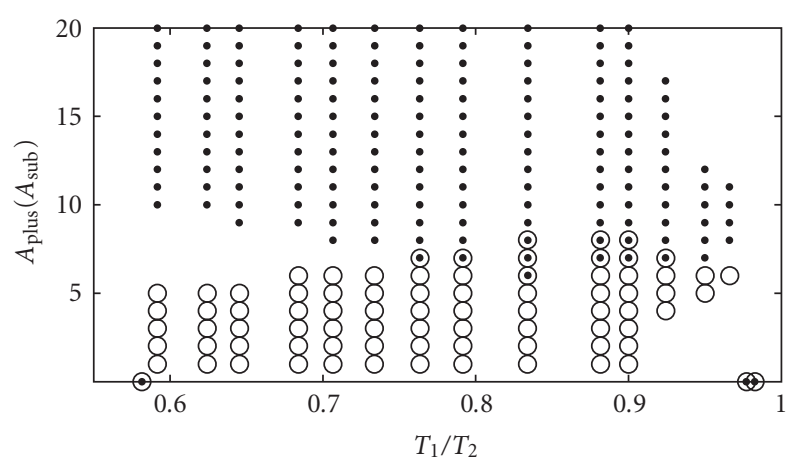

(a)

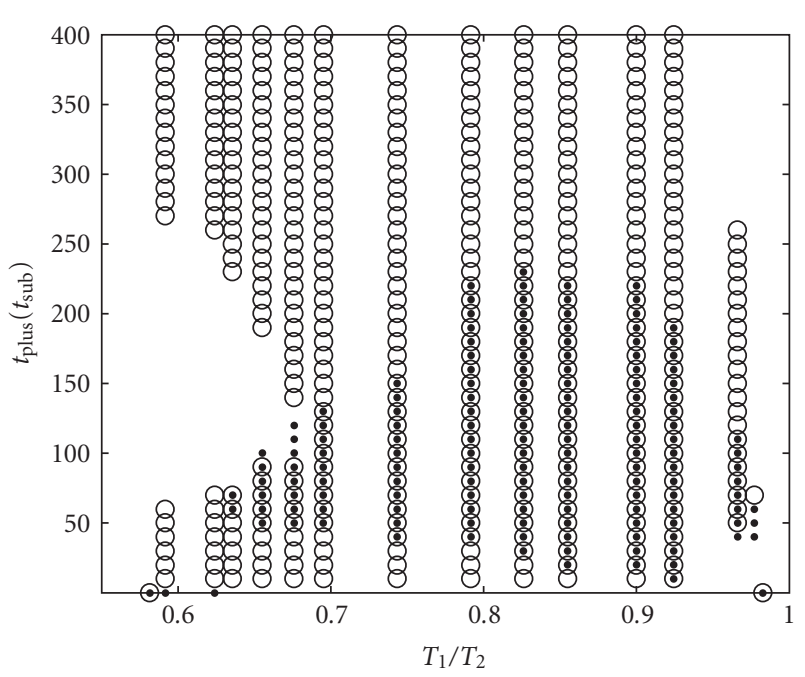

(b)

FIGURE 5: The range of learning parameters leading to synchronization for all 40 stimulations for c-STDP is presented. The points are the range of $A_{\text {plus }}$ and $t_{\text {plus }}$ which can make neuron synchronization. The circles are the range of $A_{\text {sub }}$ and $t_{\text {sub. }}$. We choose some values of $T_{2}$ to investigate the effect of $A_{\text {plus }}, A_{\text {sub }}, t_{\text {plus }}$ and $t_{\text {sub }}$ on global synchronization. $A_{\text {plus }}$ and $A_{\text {sub }}$ vary from $1 \mathrm{nS}$ to $20 \mathrm{nS}$. Let $t_{\text {plus }}$ and $t_{\text {sub }}$ vary from 10 milliseconds to 400 milliseconds. There are some values of $T_{1} / T_{2}$ marked on the horizontal axis that cannot be entrained to achieve synchronization in Figures 5(a) and 5(b).

TABLE 1: The parameters for optimal synchronization windows.

\begin{tabular}{lcccc}
\hline STDP rule & $A_{\text {plus }}(\mathrm{nS})$ & $A_{\text {sub }}(\mathrm{nS})$ & $t_{\text {plus }}(\mathrm{ms})$ & $t_{\text {sub }}(\mathrm{ms})$ \\
\hline c-STDP & 9 & 6 & 100 & 270 \\
dc-STDP & 9 & 6 & 100 & 50 \\
dc-aSTDP & 9 & 6 & 100 & 350 \\
in-STDP & 8 & 8 & 100 & 200 \\
\hline
\end{tabular}

synapse $g=25 \mathrm{nS}$. Consequently, the reported important role of STDP in synchronization should be dependent on learning parameters.

For the first three STDP rules, the optimal synchronization windows are got by regulating $t_{\text {sub }}$ while other three fixed learning parameters keep initial values. For the last STDP rule, the initial values are the optimal parameters. 


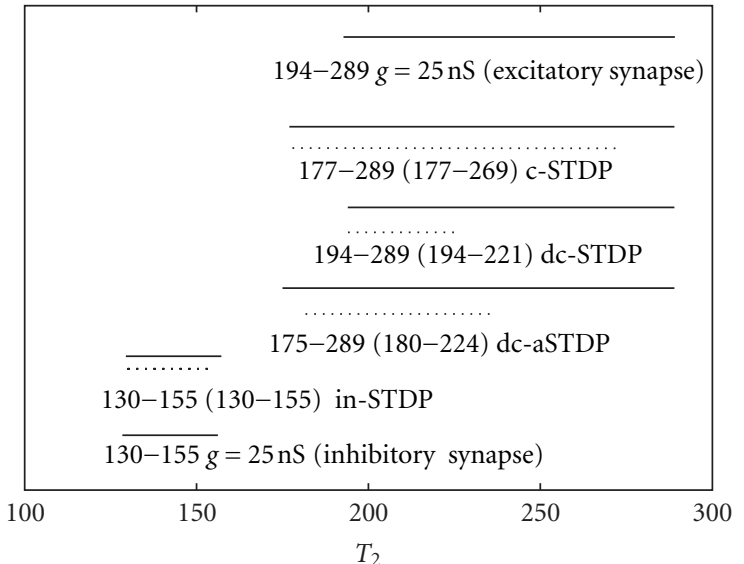

Figure 6: We present the optimal synchronization window for various learning rules compared with Figure 3. The top and the bottom lines of this figure are the synchronization windows of excitatory and inhibitory constant synapse, respectively. In addition, there are four pair lines in the middle panel for STDP rules. Each pair includes optimal synchronization window (top line) and previous synchronization window in Figure 3 (bottom line). The left range is optimal synchronization range and the range in round bracket is previous synchronization range.

3.3. The Synchronization Mechanism. It is important to understand the properties which neural synchronization depends on. We take into account this problem from two aspects.

On the one hand, synchronization correlates with the chosen STDP rules. For excitatory synapse, we find that the optimal synchronization windows for c-STDP rule and dcaSTDP rule are almost equal, and are wider than constant synapses. However, comparing with these two rules, the optimal synchronization window for dc-STDP rule is much narrower. For inhibitory STDP, the synchronization window is the same as that for constant synapse under connection strength $g=25 \mathrm{nS}$.

Accordingly, what makes the optimal synchronization windows for various STDP rules different deserves an explanation. The stationary synaptic conductance is a necessary condition for stationary synchronized state [31]. We find that the mechanisms of synchronization caused by c-STDP (or dc-aSTDP) and dc-STDP (or in-STDP) are different in our model.

Figures 7(a) and 7(b) show the average $\Delta t=t_{\text {postspike }}-$ $t_{\text {prespike, }}$ and synaptic strength after an episode of coupling time for c-STDP rule. Parameters in Figure 7 are same as those in Figure 3. There are two types of behavior for $\Delta t$ when synchronization occurs (Figure 7(a)). In a section of constant $\Delta t$, the synaptic strength does not achieve the maximal value. Apparently, in this situation, postsynaptic neuron achieves synchronization with the pre-synaptic neuron depending on the balance between potentiation and depression of synaptic conductance. In the rest part of synchronization window, the synaptic strength achieves the maximal value (Figure 7(b)). It indicates that for larger $T_{2}$, postsynaptic neuron achieves synchronization depending on the effect of maximal synaptic conductance. For dc-aSTDP rule, the synchronization mechanisms are similar to c-STDP rule. When the post-synaptic neuron synchronizes with the pre-synaptic neuron under small $T_{2}$, the change of synaptic potentiation and depression cancel each other. However, for the small portion of synchronization window at the right side, synaptic conductance gets the maximum at the stationary synchronized state.

For dc-STDP and in-STDP rules, the synchronization mechanisms may be different with the above two STDP rules. Because the potentiation and depression of synaptic conductance cancel each other, $\Delta t$ must be a fixed value for the selected STDP rule. However, for the dc-STDP rule (Figures $7(\mathrm{c})$ and $7(\mathrm{~d})), \Delta t$ keeps varying which means that the potentiation and depression of synaptic conductance do not cancel each other at the synchronization state. But, it is easily found that the synaptic conductance is at the stationary maximum for dc-STDP rule. Thus, postsynaptic neuron achieves synchronization completely depending on the effect of the maximal synaptic conductance for dc-STDP rule. The state of in-STDP rule is similar to dc-STDP rule.

As a result, neural synchronization mechanism can be different for various STDP rules. For the few $T_{2}$ at the right side of the synchronization window, the synaptic conductance achieves the maximum with c-STDP and dc-aSTDP rules. This result is obvious. Because the frequency mismatch is larger, the synapse needs to be stronger to entrain the post-synaptic neuron. But for most part of synchronization window at the left, c-STDP and dc-aSTDP rules rely on the balance of potentiation and depression. Instead of balancing out potentiation and depression during one cycle, dc-STDP and in-STDP rules depend on synaptic strength achieving its maximum. It is important to understand why c-STDP and dc-aSTDP rely on the balance of potentiation and depression while dc-STDP and in-STDP do not.

Nowotny et al. have introduced the mechanisms behind the enhancement of neural synchronization by c-STDP rule which rely on the balance of potentiation and depression. The synapse strength remains stable regardless of postsynaptic neuron firing later or earlier attributed to the specific shape of c-STDP curve. The situation of c-STDP is similar to dc-aSTDP. We adopt the similar analysis method [31] here for the dc-aSTDP and dc-STDP. The time lags are recorded as $\Delta t_{1}$ and $\Delta t_{2}$, where $\Delta t_{1}-\Delta t_{2}=T_{1}=T_{2}{ }^{1}$ and $\Delta g_{1}-\Delta g_{2}=0$ (Figure 8) at this state. If post-synaptic neuron fires faster, $\Delta t_{1}$ becomes smaller. Synaptic strength will be depressed, due to $\Delta g_{1}-\Delta g_{2}<0$ for dc-aSTDP rule, so that the post-synaptic neuron is less excited and goes back into the synchronized state (Figure $8(\mathrm{~b})$ ). The other direction can be analyzed in the same way for dc-aSTDP. But for dc-STDP, when post-synaptic neuron fires faster, synaptic strength will be increased due to $\Delta g_{1}-\Delta g_{2}>0$ in this case. The post-synaptic neuron is more excited and cannot go back into the synchronized state (Figure $8(\mathrm{c})$ ). The opposite direction is the same case for dc-STDP and cannot go back into the synchronized state. Therefore, the synchronization mechanisms between these two rules are different.

For in-STDP rule, we can easily find that $\Delta g_{1}-\Delta g_{2}$ is always positive, where $\Delta g_{1}-\Delta g_{2}=A_{\text {plus }} *\left(\exp \left(-x / t_{\text {plus }}\right)-\right.$ $0.5)-A_{\text {sub }} *\left(\exp \left(x-171 / t_{\text {sub }}\right)-0.5\right)$ and values of parameters 


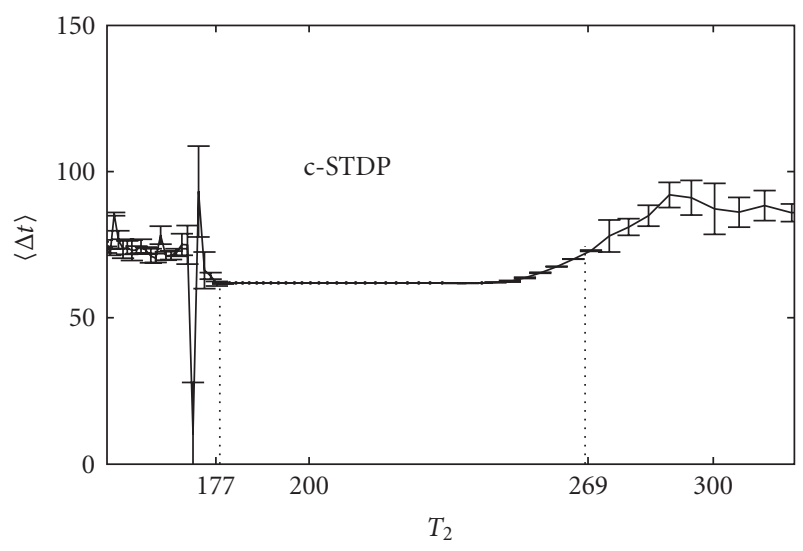

(a)

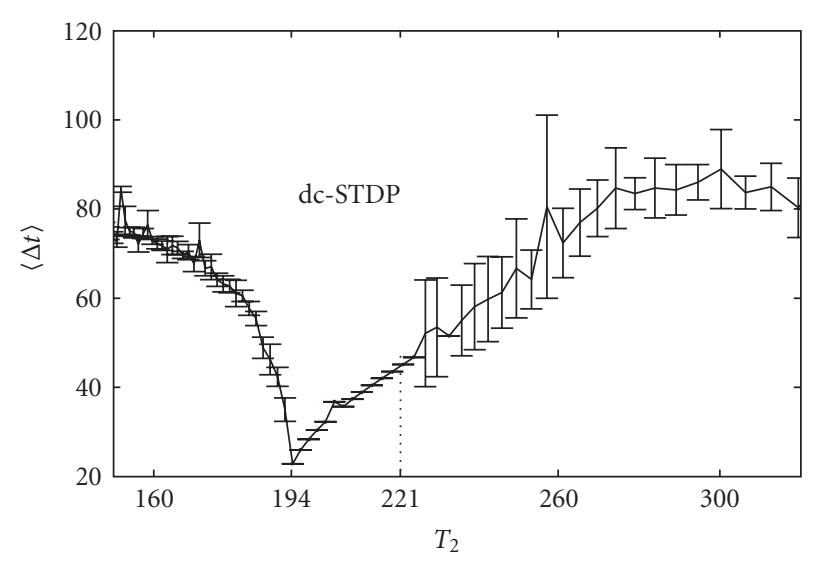

(c)

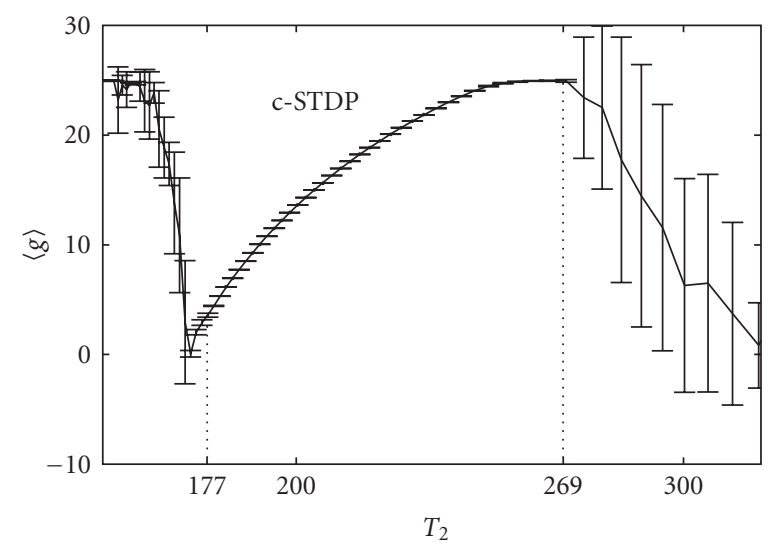

(b)

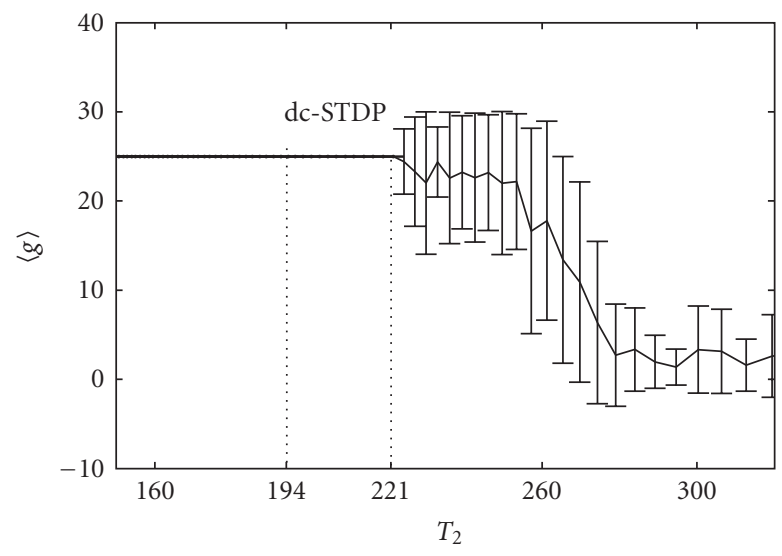

(d)

FIGURE 7: Four pictures are obtained from the same values of parameters and model of Figure 3. The front four pictures take the function of tanh to limit synaptic strength for c-STDP and dc-STDP rules. Figures 7(a) and 7(c) show the average spike time interval of postneuron's and preneuron's spike time for c-STDP and dc-STDP over some time after a period time of coupling, respectively. Figures 7(b) and 7(d) present the average synaptic strength for c-STDP and dc-STDP rule, respectively. Each subplot has two dash lines what indicate the boundary of synchronization window.

are the same as Figure 3. It means that the potentiation and depression of synaptic conductance during one period cannot achieve balance. The synaptic strength must achieve the maximum resulting from $\Delta g_{1}-\Delta g_{2}>0$.

On the other hand, learning parameters also play important role in neural synchronization. We try to explain the role of a learning parameter by considering how it influences the synapse conductance, which is a major factor for synchronizing neurons with a given mismatch of intrinsic frequencies.

It is obvious that if synaptic conductance becomes stronger, it can make larger $T_{2}$ to achieve the same period with pre-synaptic neuron for various STDP rules. Thus, with other three learning parameters fixed, larger $A_{\text {plus }}$ values, which are corresponding to the stronger stable synaptic strength for c-STDP rule, can cause larger $T_{2}$ synchronization. Similarly, smaller $A_{\text {sub }}$ values will give rise to larger $T_{2}$ synchronization for c-STDP (Figure 5(a)). Moreover, from Figure 5(b), moderate $t_{\text {plus }}$ values can also make larger $T_{2}$ synchronized to $T_{1}$ because these values bring about stronger synaptic strength. We can prove this perspective by simple calculus reasoning. Based on the expression of c-STDP rule in Section 2, let $t_{\text {plus }}$ be variable and let other parameters keep constant. $\Delta g_{\text {raw }}$ is viewed as the function of variable $t_{\text {plus }}$. By calculating the derivative of $\Delta g_{\text {raw }}$, we can find that $\Delta g_{\text {raw }}$ is a first increasing and then decreasing function when $t_{\text {plus }}$ increases gradually. Thus, medial values of $t_{\text {plus }}$ can result in stronger synaptic strength. By the same reasoning for $t_{\text {sub, we }}$ can conclude that smaller or larger $t_{\text {sub }}$ can make larger $T_{2}$ synchronization for c-STDP (Figure 5(b)).

The effects of learning parameters on synchronization about other learning rules are similar to the c-STDP rule for larger $T_{2}$. However, smaller $T_{2}$ values leading to synchrony are only related to c-STDP and dc-aSTDP, because they rely on the balance of depression and potentiation which could lead to an appropriate low stable synaptic strength. Therefore, only proper learning parameters got by regulating the effect of learning parameters on synchronization are required for smaller $T_{2}$ achieved synchronization.

Finally, we conclude why the widest synchronization windows for some STDP rules are different. From the above statements, we find that two aspects affect synchronization. 


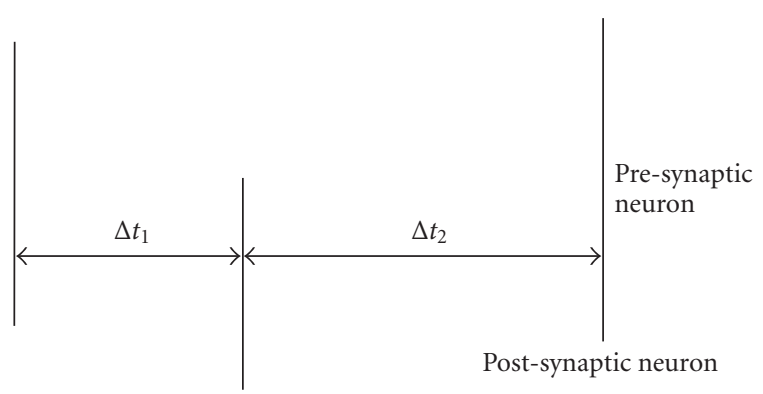

(a)
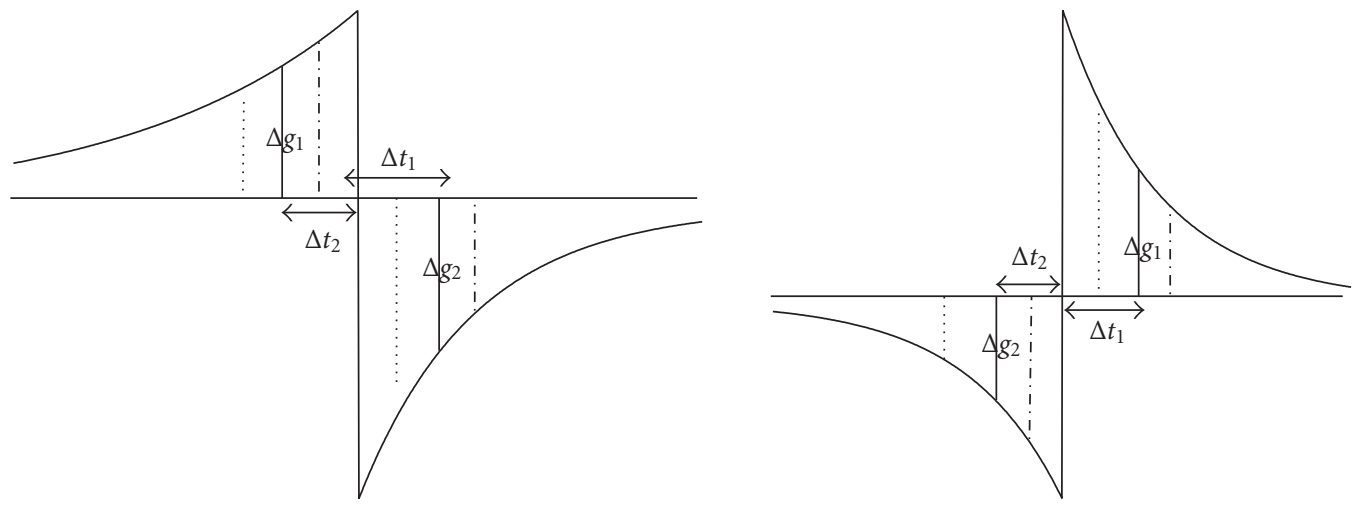

\footnotetext{
- $\Delta g_{1}-\Delta g_{2}=0$, no net change in conductance

..... The post-synaptic neuron fires faster

...- The post-synaptic neuron fires slower
}

(b)

\footnotetext{
- $\Delta g_{1}-\Delta g_{2}=0$, no net change in conductance

..... The post-synaptic neruon fires faster

-.- The post-synaptic neuron fires slower
}

(c)

FIGURE 8: Different synchronization mechanisms for different STDP rules. (a) Shows the situation of $\Delta t_{1}-\Delta t_{2}=T_{1}=T_{2}$, where $\Delta g_{1}-\Delta g_{2}=$ 0 . The solid lines in (b) and (c) are same as (a). (b) and (c) present the change of synaptic strength of dc-aSTDP and dc-STDP rules, respectively.

One is the maximum synaptic strength which can make larger $T_{2}$ synchronize. The other is the balance of depression and potentiation which can make smaller and moderate $T_{2}$ synchronize. For c-STDP and dc-aSTDP, they can achieve the widest synchronization windows through modulating the two aspects. However, dc- STDP and in- STDP rules, due to their specific shape, can only make use of the first one. This implies that the widest synchronization windows for dcSTDP and in-STDP rules cannot exceed the synchronization windows for the maximum constant connection strength under the same model respectively. Therefore, the optimal synchronization windows for c-STDP and dc-aSTDP are wider than those for dc-STDP and in-STDP.

\section{Conclusion}

STDP plays important functional role in neural synchronization. The mechanism of STDP in neuronal synchronization is still not completely clear. Inspired by previous experiments and theoretical researches, we study the important aspects of STDP-induced synchronization in this paper, such as the role of various STDP rule in synchronization, the widest synchronization window through regulating learning parameters, and synchronization mechanism.
In order to explore the functional role of STDP in synchronization, we compare synchronization windows of different types of STDP rules with that of constant synapse under the same model parameters. For the given parameters, not all synchronization windows are enhanced by STDP rules.

Synchronized responses have a stronger influence on cells at subsequent processing stages than nonsynchronized responses [48-50]. And the enhanced precise synchronization is important in improving a rapid and reliable transmission of information about sensory changes $[2,51]$. Recent researches have reported various methods to enhance synchronization, such as, selective attention [52] and time delay [53]. Here, we present the effect of modulation of learning parameters on synchronization and optimal synchronization window which are not narrower than constant synapse. The optimal synchronization windows by c-STDP and dc-aSTDP rules are much wider than constant synapse. It indicates that the function role of STDP rule in synchronization depends on the learning parameters.

The synchronization mechanism is also described here. Different shapes of STDP rule can cause different optimal synchronization windows. The optimal synchronization windows of c-STDP and dc-aSTDP are wider than that of 
dc-STDP for excitatory synapse. For c-STDP rule and dcaSTDP rule, a stationary synchronized state completely depends on the balance between potentiation and depression or the maximal synaptic conductance. However, for dc-STDP and in-STDP rule, the stable synchronized state depends on the maximal synaptic conductance under the self-limitation of synaptic strength. If we change the type of bound of synaptic strength for dc-STDP rule from self-limitation to artificial bounds, we find that the synchronization mechanism does not change. In a word, on one hand, the synchronization range of dc-aSTDP and c-STDP can achieve the optimal synchronization window of dc-STDP, depending on the maximal synaptic conductance. On the other hand, dcaSTDP and c-STDP can extend the synchronization windows to include smaller $T_{2}$ by the balance between potentiation and depression.

The firing pattern of neurons is regular in this paper. Many neurons in brain areas present regular firing. For example, neurons in cat area 17 can be grouped in 4 different electrophysiological cell classes, including regular spiking [54]. And spontaneous, regular action potentials were observed both with cell-attached patch recordings as well as with whole cell current-clamp recordings for cholinergic neurons in the parabigeminal nucleus of the rat midbrain [55]. Neuronal synchronization properties with regular firing neurons have been studied. For example, whether pyramidal neurons in different cortical layers exhibit similar tendencies to synchronize is studied [56]. Based on this point and the functional role of STDP in synchronization, we explore the synchronization windows of various STDP rules from the view of neurons' regular firing.

STDP-mediated synchronization is a remarkably robust phenomenon against strong noise [30, 31]. Although our simulation is not under the noise environment, our results may represent some predictions for STDP-mediated synchronization in noisy environment. In addition, from Figure 2(a), we can clearly see that some synchronization number is between 1 and 39. We estimate that it may be related to phase.

Our results present that the range of $T_{2}$ values leading to synchrony increases strongly if the constant synaptic connection is increased from $12.5 \mathrm{nS}$ to $25 \mathrm{nS}$. Nowotny et al. find that the extent of synchrony does not change considerably by doubling the synaptic conductance [31]. Their result is not conflict with our result. There are three parameters that are different, $V_{\text {slope }} t_{\text {syn }}$, and $g_{\text {max }}$. Furthermore, $T_{1}$ is fixed at 171 milliseconds in our simulation while $T_{2}$ is fixed at 300 milliseconds in theirs. When $T_{2}$ is fixed, the range of $T_{1}$ values leading to synchrony is limited from 0 to $T_{2}$ no matter how the strength of constant connection changes. However, when $T_{1}$ is fixed, the range of $T_{2}$ values leading to synchrony can vary from $T_{1}$ to very large value due to the increase of the constant connection. At the same time, if we adopt the same parameters with Nowotny's paper, the similar result can be got. Furthermore, when constant synaptic connection is $0 \mathrm{nS}$, it is clear that two neurons with different initial periods cannot synchronize. This situation means that the length of synchronization window is 0 . Along with the increase of strength of constant connection, some $T_{2}$ must cause the synchronization. Thus, it is easily found that synchronization window must become wider by increasing the constant connection to some degree.

We mainly discuss synchronization for different STDP rules in this paper. The question of how the time windows of various STDP rules are biophysically regulated remains relatively unexplored. There are some experiments using neuromodulators to study the time window for STDP [57]. We are interested in building molecular kinetic equations for STDP to explain our results.

It has been proposed that conscious perception depends on the transient synchronization of widely distributed neural assemblies [58]. And long-distance synchronization plays a role in triggering the cognitive processes associated with conscious awareness [59]. The changed learning parameters by neuromodulators may influence the cognitive processes. In addition, some diseases and the function of brain are related with synchronization mentioned above, especially in theta $(4-8 \mathrm{~Hz})$ rhythm synchronization during fear memory retrieval [43] which is consistent with what we considered here. Therefore, our work may advance understanding of synchronization to some extent. And we expect that our simulation results will provide some help for related diseases treatment.

\section{Acknowledgments}

This research is supported by the Educational Department of Jiangxi Province of China (GJJ08475) and Jiangxi Blue Sky University (XYKJ08YB05).

\section{References}

[1] J. Fell, P. Klaver, K. Lehnertz, et al., "Human memory formation is accompanied by rhinal-hippocampal coupling and decoupling," Nature Neuroscience, vol. 4, no. 12, pp. 12591264, 2001.

[2] T. Womelsdorf, P. Fries, P. P. Mitra, and R. Desimone, "Gamma-band synchronization in visual cortex predicts speed of change detection," Nature, vol. 439, no. 7077, pp. 733-736, 2006.

[3] L. Melloni, C. Molina, M. Pena, D. Torres, W. Singer, and E. Rodriguez, "Synchronization of neural activity across cortical areas correlates with conscious perception," Journal of Neuroscience, vol. 27, no. 11, pp. 2858-2865, 2007.

[4] T. Womelsdorf and P. Fries, "Neuronal coherence during selective attentional processing and sensory-motor integration," Journal of Physiology, vol. 100, no. 4, pp. 182-193, 2006.

[5] J. Biederlack, M. Castelo-Branco, S. Neuenschwander, D. W. Wheeler, W. Singer, and D. Nikolić, "Brightness induction: rate enhancement and neuronal synchronization as complementary codes," Neuron, vol. 52, no. 6, pp. 1073-1083, 2006.

[6] M. Chavez, M. Le Van Quyen, V. Navarro, M. Baulac, and J. Martinerie, "Spatio-temporal dynamics prior to neocortical seizures: amplitude versus phase couplings," IEEE Transactions on Bio-Medical Engineering, vol. 50, no. 5, pp. 571-583, 2003.

[7] C. M. T. Queiroz and L. E. Mello, "Synaptic plasticity of the CA3 commissural projection in epileptic rats: an in vivo electrophysiological study," European Journal of Neuroscience, vol. 25, no. 10, pp. 3071-3079, 2007.

[8] H. Liang, S. L. Bressler, M. Ding, R. Desimone, and P. Fries, "Temporal dynamics of attention-modulated neuronal 
synchronization in macaque V4," Neurocomputing, vol. 52-54, pp. 481-487, 2003.

[9] A. D. Wagner, "Synchronicity: when you're gone I'm lost without a trace?" Nature Neuroscience, vol. 4, no. 12, pp. 1159 1160, 2001.

[10] N. Axmacher, F. Mormann, G. Fernández, C. E. Elger, and J. Fell, "Memory formation by neuronal synchronization," Brain Research Reviews, vol. 52, no. 1, pp. 170-182, 2006.

[11] F. Varela, J.-P. Lachaux, E. Rodriguez, and J. Martinerie, "The brainweb: phase synchronization and large-scale integration," Nature Reviews Neuroscience, vol. 2, no. 4, pp. 229-239, 2001.

[12] T. V. P. Bliss and T. Lomo, "Long lasting potentiation of synaptic transmission in the dentate area of the anaesthetized rabbit following stimulation of the perforant path," The Journal of Physiology, vol. 232, no. 2, pp. 331-356, 1973.

[13] D. J. Linden and J. A. Connor, "Long-term synaptic depression," Annual Review of Neuroscience, vol. 18, no. 1, pp. 319 357, 1995.

[14] R. A. Nicoll and R. C. Malenka, "Contrasting properties of two forms of long-term potentiation in the hippocampus," Nature, vol. 377, no. 6545, pp. 115-118, 1995.

[15] W. Gerstner and J. L. van Hemmen, "Coding and information processing in neural networks," in Models of Neural Networks II: Temporal Aspects of Coding and Information Processing in Biological Systems, 1994.

[16] W. Gerstner, R. Kempter, J. L. Van Hemmen, and H. Wagner, "A neuronal learning rule for sub-millisecond temporal coding," Nature, vol. 383, no. 6595, pp. 76-78, 1996.

[17] H. Markram, J. Lübke, M. Frotscher, and B. Sakmann, "Regulation of synaptic efficacy by coincidence of postsynaptic APs and EPSPs," Science, vol. 275, no. 5297, pp. 213-215, 1997.

[18] D. Debanne, B. H. Gahwiler, and S. M. Thompson, "Longterm synaptic plasticity between pairs of individual CA3 pyramidal cells in rat hippocampal slice cultures," The Journal of Physiology, vol. 507, no. 1, pp. 237-247, 1998.

[19] G.-Q. Bi and M.-M. Poo, "Synaptic modifications in cultured hippocampal neurons: dependence on spike timing, synaptic strength, and postsynaptic cell type," Journal of Neuroscience, vol. 18, no. 24, pp. 10464-10472, 1998.

[20] L. I. Zhang, H. W. Tao, C. E. Holt, W. A. Harris, and M.M. Poo, "A critical window for cooperation and competition among developing retinotectal synapses," Nature, vol. 395, no. 6697, pp. 37-44, 1998.

[21] W. Gerstner, R. Ritz, and J. L. van Hemmen, "Why spikes? Hebbian learning and retrieval of time-resolved excitation patterns," Biological Cybernetics, vol. 69, no. 5-6, pp. 503-515, 1993.

[22] A. A. Minai and W. B. Levy, "Sequence learning in a single trial," in Proceedings of the INNS World Congress on Neural Networks II, pp. 505-508, 1993.

[23] L. F. Abbott and K. I. Blum, "Functional significance of long-term potentiation for sequence learning and prediction," Cerebral Cortex, vol. 6, no. 3, pp. 406-416, 1996.

[24] H. Watanabe, M. Watanabe, K. Aihara, and S. Kondo, "Change of memory formation according to STDP in a continuoustime neural network model," Systems and Computers in Japan, vol. 35, no. 12, pp. 57-66, 2004.

[25] W. Gerstner, R. Kempter, et al., "A developmental learning rule for coincidence tuning in the barn owl auditory system," in Proceedings of the annual conference on Computational Neuroscience: Trends in Research, J. Bower, Ed., vol. 665, pp. 665-669, Plenum Presss, New York, NY, USA, 1997.
[26] K. I. Blum and L. F. Abbott, "A model of spatial map formation in the hippocampus of the rat," Neural Computation, vol. 8, no. 1, pp. 85-93, 1996.

[27] M. R. Mehta, M. C. Quirk, and M. A. Wilson, "Experiencedependent asymmetric shape of hippocampal receptive fields," Neuron, vol. 25, no. 3, pp. 707-715, 2000.

[28] M. R. Mehta and M. A. Wilson, "From hippocampus to V1: effect of LTP on spatio-temporal dynamics of receptive fields," Neurocomputing, vol. 32-33, pp. 905-911, 2000.

[29] T.-C. Chao and C.-M. Chen, "Learning-induced synchronization and plasticity of a developing neural network," Journal of Computational Neuroscience, vol. 19, no. 3, pp. 311-324, 2005.

[30] V. P. Zhigulin, M. I. Rabinovich, R. Huerta, and H. D. I. Abarbanel, "Robustness and enhancement of neural synchronization by activity-dependent coupling," Physical Review E, vol. 67, no. 2, Article ID 021901, 4 pages, 2003.

[31] T. Nowotny, V. P. Zhigulin, A. I. Selverston, H. D. I. Abarbanel, and M. I. Rabinovich, "Enhancement of synchronization in a hybrid neural circuit by spike-timing dependent plasticity," Journal of Neuroscience, vol. 23, no. 30, pp. 9776-9785, 2003.

[32] R. D. Traub and R. Miles, Neuronal Networks of the Hippocampus, Cambridge University Press, Cambridge, UK, 1991.

[33] R. Kempter, W. Gerstner, and J. L. Van Hemmen, "Intrinsic stabilization of output rates by spike-based Hebbian learning," Neural Computation, vol. 13, no. 12, pp. 2709-2741, 2001.

[34] S. Song and L. F. Abbott, "Cortical development and remapping through spike timing-dependent plasticity," Neuron, vol. 32, no. 2, pp. 339-350, 2001.

[35] P. D. Roberts and C. C. Bell, "Spike timing dependent synaptic plasticity in biological systems," Biological Cybernetics, vol. 87, no. 5-6, pp. 392-403, 2002.

[36] L. F. Abbott and S. B. Nelson, "Synaptic plasticity: taming the beast," Nature Neuroscience, vol. 3, supplement, pp. 1178$1183,2000$.

[37] C. C. Bell, V. Z. Han, Y. Sugawara, and K. Grant, "Synaptic plasticity in a cerebellum-like structure depends on temporal order," Nature, vol. 387, no. 6630, pp. 278-281, 1997.

[38] D. K. Bilkey and U. Heinemann, "Intrinsic theta-frequency membrane potential oscillations in layer III/V perirhinal cortex neurons of the rat," Hippocampus, vol. 9, no. 5, pp. 510$518,1999$.

[39] H.-C. Pape, R. T. Narayanan, J. Smid, O. Stork, and T. Seidenbecher, "Theta activity in neurons and networks of the amygdala related to long-term fear memory," Hippocampus, vol. 15, no. 7, pp. 874-880, 2005.

[40] L. Aftanas and S. Golosheykin, "Impact of regular meditation practice on EEG activity at rest and during evoked negative emotions," International Journal of Neuroscience, vol. 115, no. 6, pp. 893-909, 2005.

[41] D. Paré and D. R. Collins, "Neuronal correlates of fear in the lateral amygdala: multiple extracellular recordings in conscious cats," Journal of Neuroscience, vol. 20, no. 7, pp. 2701-2710, 2000.

[42] D. Paré, D. R. Collins, and J. G. Pelletier, "Amygdala oscillations and the consolidation of emotional memories," Trends in Cognitive Sciences, vol. 6, no. 7, pp. 306-314, 2002.

[43] T. Seidenbecher, T. R. Laxmi, et al., in Amygdalar and Hippocampal Theta Rhythm Synchronization during Fear Memory Retrieval, vol. 301, pp. 846-850, American Association for the Advancement of Science, 2003.

[44] T. Aoki and T. Aoyagi, "Synchrony-induced switching behavior of spike pattern attractors created by spike-timingdependent plasticity," Neural Computation, vol. 19, no. 10, pp. 2720-2738, 2007. 
[45] E. D. Lumer, Effects of Spike Timing on Winner-Take-All Competition in Model Cortical Circuits, vol. 12, MIT Press, Cambridge, Mass, USA, 2000.

[46] T. Aoyagi and T. Aoki, "Possible role of synchronous input spike trains in controlling the function of neural networks," Neurocomputing, vol. 58-60, pp. 259-264, 2004.

[47] T. Akimitsu, Y. Okabe, and A. Hirose, "Self-organization through spike-timing dependent plasticity using localized synfire-chain patterns," Neural Processing Letters, vol. 25, no. 1, pp. 79-89, 2007.

[48] J.-M. Alonso, W. M. Usrey, and R. C. Reid, "Precisely correlated firing in cells of the lateral geniculate nucleus," Nature, vol. 383, no. 6603, pp. 815-819, 1996.

[49] M. Brecht, W. Singer, and A. K. Engel, "Correlation analysis of corticotectal interactions in the cat visual system," Journal of Neurophysiology, vol. 79, no. 5, pp. 2394-2407, 1998.

[50] W. Singer, "Neuronal synchrony: a versatile code for the definition of relations?" Neuron, vol. 24, no. 1, pp. 49-65, 1999.

[51] M. Diesmann, M.-O. Gewaltig, and A. Aertsen, "Stable propagation of synchronous spiking in cortical neural networks," Nature, vol. 402, no. 6761, pp. 529-533, 1999.

[52] P. Fries, T. Womelsdorf, R. Oostenveld, and R. Desimone, "The effects of visual stimulation and selective visual attention on rhythmic neuronal synchronization in macaque area V4," Journal of Neuroscience, vol. 28, no. 18, pp. 4823-4835, 2008.

[53] Q.-Y. Wang and Q.-S. Lu, "Time delay-enhanced synchronization and regularization in two coupled chaotic neurons," Chinese Physics Letters, vol. 22, no. 3, pp. 543-546, 2005.

[54] L. G. Nowak, M. V. Sanchez-Vives, and D. A. McCormick, "Lack of orientation and direction selectivity in a subgroup of fast-spiking inhibitory interneurons: cellular and synaptic mechanisms and comparison with other electrophysiological cell types," Cerebral Cortex, vol. 18, no. 5, pp. 1058-1078, 2008.

[55] C. A. Goddard, E. I. Knudsen, and J. R. Huguenard, "Intrinsic excitability of cholinergic neurons in the rat parabigeminal nucleus," Journal of Neurophysiology, vol. 98, no. 6, pp. 34863493, 2007.

[56] Y. Tsubo, M. Takada, A. D. Reyes, and T. Fukai, "Layer and frequency dependencies of phase response properties of pyramidal neurons in rat motor cortex," European Journal of Neuroscience, vol. 25, no. 11, pp. 3429-3441, 2007.

[57] G. H. Seol, J. Ziburkus, S. Huang, L. Song, I. T. Kim, and K. Takamiya, "Neuromodulators control the polarity of spiketiming-dependent synaptic plasticity," Neuron, vol. 55, no. 6, pp. 919-929, 2007.

[58] E. Thompson and F. J. Varela, "Radical embodiment: neural dynamics and consciousness," Trends in Cognitive Sciences, vol. 5, no. 10, pp. 418-425, 2001.

[59] S. Dehaene, J.-P. Changeux, L. Naccache, J. Sackur, and C. Sergent, "Conscious, preconscious, and subliminal processing: a testable taxonomy," Trends in Cognitive Sciences, vol. 10, no. 5, pp. 204-211, 2006. 

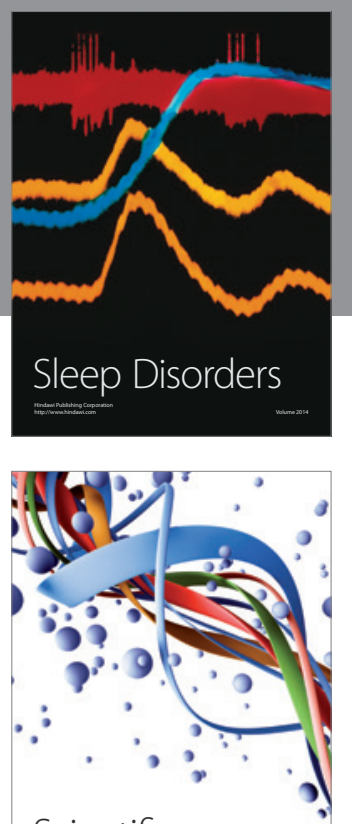

Scientifica
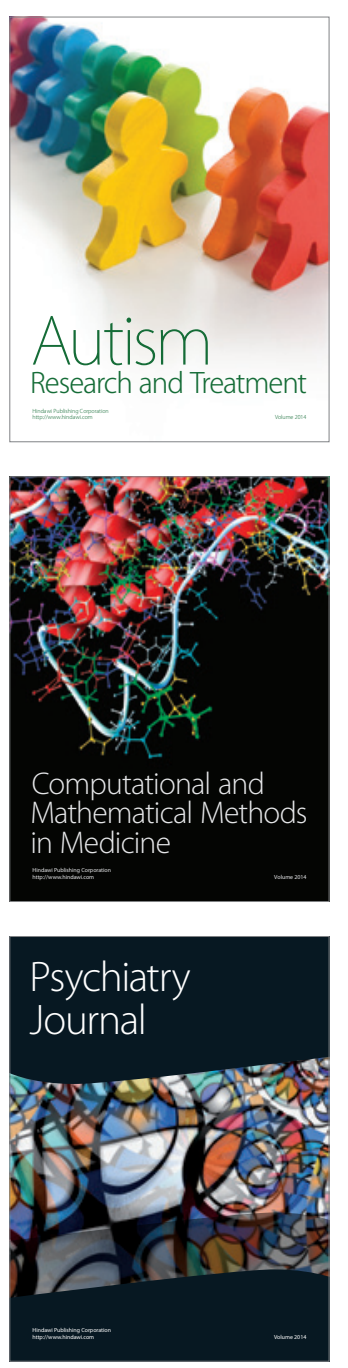
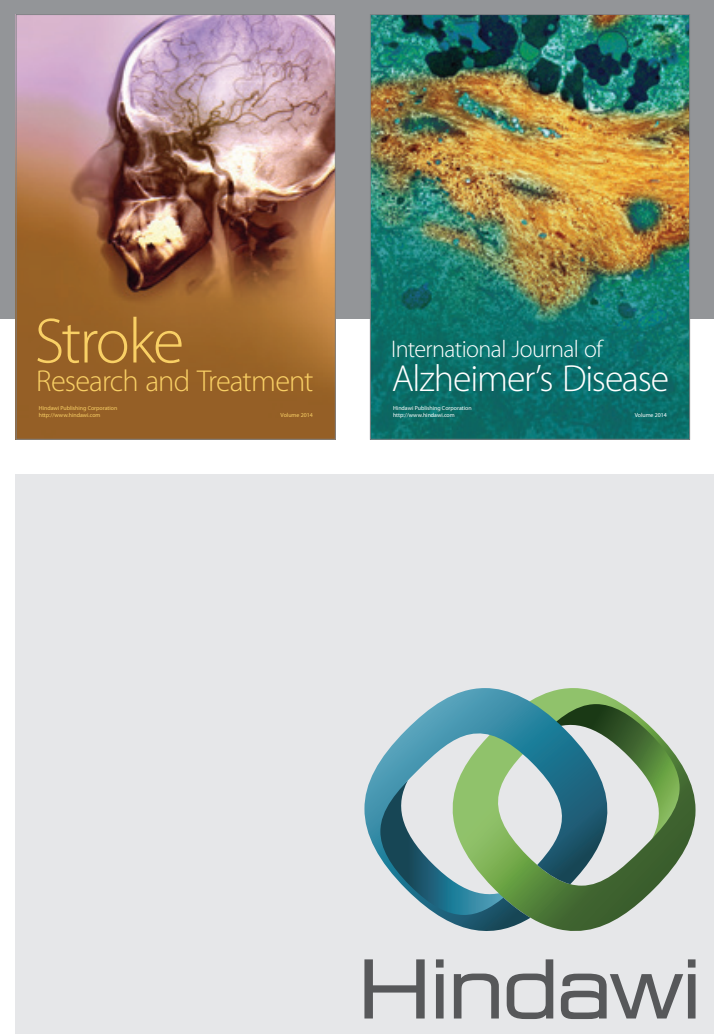

Submit your manuscripts at

http://www.hindawi.com
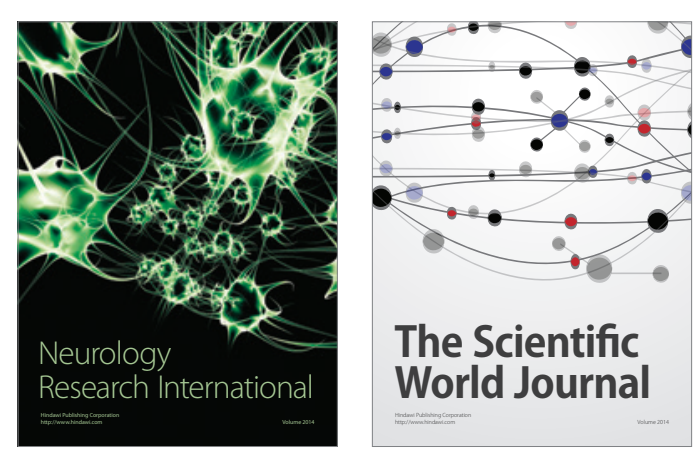

The Scientific World Journal

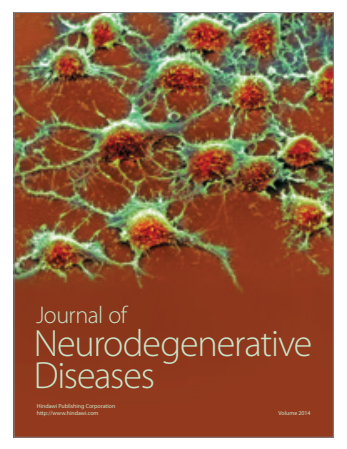

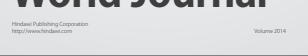

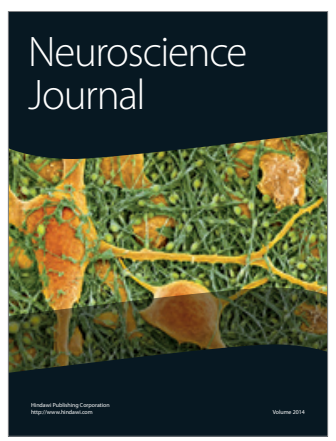

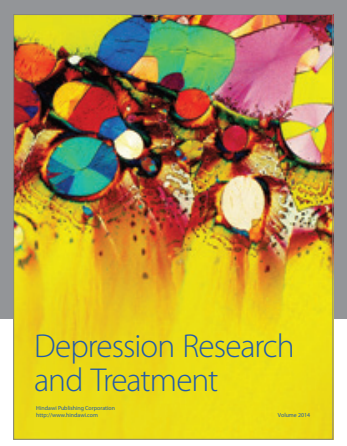
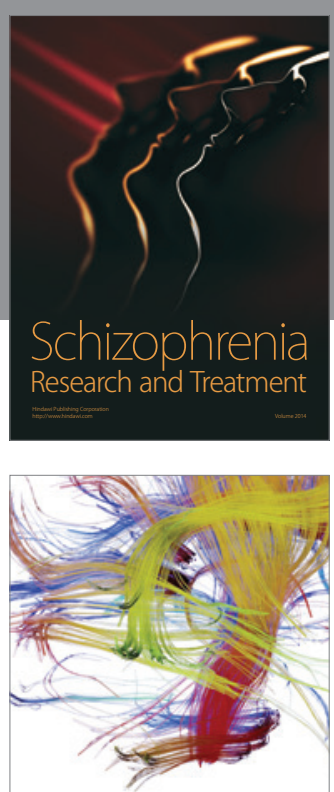

Brain Science

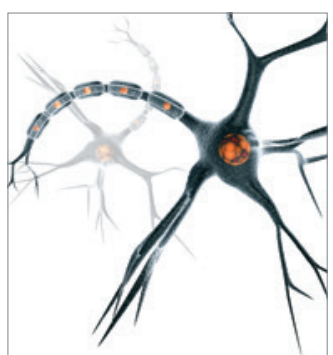

Neural Plasticity
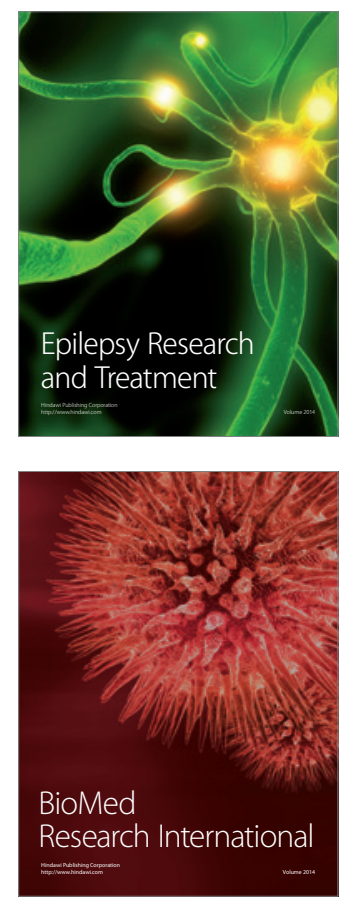

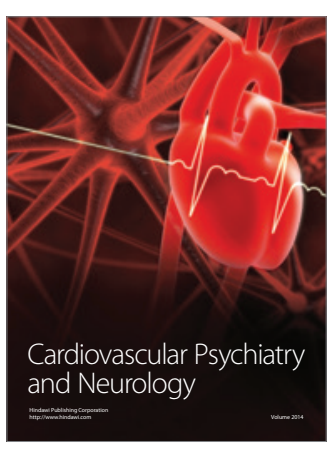

Parkinson's

Disease
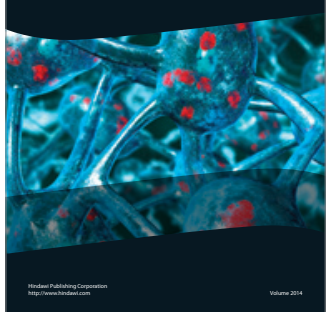